\title{
Pifithrin- $\mu$ is efficacious against non-small cell lung cancer via inhibition of heat shock protein 70
}

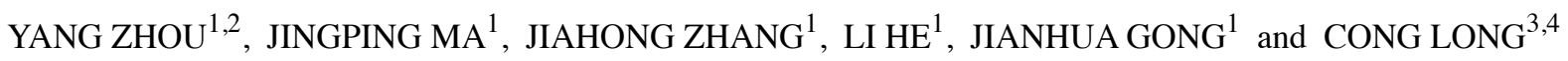 \\ ${ }^{1}$ Department of Respiratory Medicine, The Second Clinical Medical College, Yangtz University; \\ ${ }^{2}$ Department of Respiratory Medicine, Jingzhou Hospital, Tongji Medical College, \\ Huazhong University of Science and Technology (HUST), Jingzhou, Hubei 434020; \\ ${ }^{3}$ Department of Laboratory Medicine, Jingzhou First People's Hospital; \\ ${ }^{4}$ Department of Laboratory Medicine, The First Clinical Medical College, \\ Yangtz University, Jingzhou, Hubei 434100, P.R. China
}

Received June 23, 2016; Accepted November 16, 2016

DOI: 10.3892/or.2016.5286

\begin{abstract}
Heat-shock protein (Hsp) 70, known as a pro-survival protein, is aberrantly expressed in several malignancies. The small molecule 2-phenylethyenesulfonamide (PES), also referred to as pifithrin- $\mu$, is known as an HSP70 inhibitor, which exhibits antitumor activities in a variety of cancer cell lines. However, little is known about its effect on non-small cell lung cancer (NSCLC) cell lines. This study aimed to investigate the effect of PES on human NSCLC cell lines A549 and $\mathrm{H} 460$, and explore the possible underlying mechanism of action. Cell viability assay by using CCK- 8 kits was performed to demonstrate that PES dose- and timedependently inhibited proliferation of A549 and H460 cells. Wound healing assay and Transwell migration assay results indicated that PES inhibited cell migration of A549 and H460 cells. Flow cytometry results demonstrated that PES resulted in G0/G1 phase cell cycle arrest, and induced apoptosis via a caspase-dependent manner in A549 and H460 cells. Western blotting results suggested that phosphorylation of AKT and ERK was inhibited by PES treatment. In addition, death receptor 4 (DR4) and DR5 were increased by PES treatment. Overexpression of Hsp70 in A549 cells attenuated the growth inhibitory efficiency of PES. Knockdown of Hsp70 in A549 cells enhanced sensitivity of PES to cell growth inhibition, suggesting that the inhibitory effect of PES on cell proliferation is specifically through Hsp70-dependent mechanism. PES and tumor necrosis factor-related apoptosis-inducing ligand
\end{abstract}

Correspondence to: Professor Cong Long, Department of Laboratory Medicine, Jingzhou First People's Hospital, 8 Hangkong Road, Jingzhou, Hubei 434100, P.R. China

E-mail: conglong@whu.edu.cn

Key words: pifithrin- $\mu$, heat shock protein 70, tumor necrosis factorrelated apoptosis-inducing ligand, tumor therapy, non-small cell lung cancer
(TRAIL) exerts a potent synergistic effect on cell proliferation inhibition and induction of apoptosis in A549 and $\mathrm{H} 460$ cells. In a mouse xenograft model of lung cancer by A549 cells, PES treatment displayed significant inhibitory effects on tumor growth. All these findings suggest that PES shows antitumor activity against human NSCLC in vitro and in vivo, and therefore may be a promising agent for use to the treatment of NSCLC.

\section{Introduction}

Lung cancer is one of the most prevalent and fatal type of cancers in the world, accounting for $\sim 20 \%$ of all cancerrelated death (1). Lung cancer can be divided into two broad categories, small cell lung cancer and non-small cell lung cancer (NSCLC). NSCLC accounting for $\sim 80 \%$ of all lung cancer cases leads to the highest mortality with a relative poor 5 -year survival rate. Although surgical and chemotherapeutic techniques have made great progress for the treatment of lung cancer, the prognosis of NSCLC is very poor due to toxicity, high incidence of recurrence, and other side effects, with a 5 -year survival rate as low as $15 \%$ (2). Therefore, it is urgent and necessary for us to explore new strategies or drugs with fewer side effects for the management of NSCLC.

Heat shock protein (Hsp) family is a group of conserved molecular chaperons that facilitate proper protein folding, modification, and transportation, and are known as inhibitors of apoptosis (3). Hsp expression usually increases in cells under stressful condition, including increased temperature, hypoxia, and exposure to cytotoxic agents, to protect cells from injury (4). Hsp70 is a member of Hsps, and Hsp70 overexpression has been reported to be associated with a wide range of malignances $(5,6)$. Increasing results have reported that compared to healthy individuals, Hsp70 has been overexpressed in serum and tissue samples from patients with $\operatorname{NSCLC}(7,8)$. Furthermore, overexpression of Hsp70 is associated with adverse prognosis and resistance to chemotherapy (5). Selective depletion of Hsp70 in lung cancer cells results in apoptotic cell death, while not in normal lung cells, suggesting 
that targeting Hsp70 may be a potential approach for cancer therapeutics (9).

Previous results identified the small molecular 2-phenylethynesulfonamide (PES), also known as pifithrin- $\mu$, as a specific inhibitor of stress-inducible Hsp70, which induced tumor cell death but markedly showed less toxic to nontransformed cells (10). Several pivotal survival pathways for cancer were impaired by PES through disruption of HSP70/ HSP90 chaperone system (11). Data reported by Granato et al indicated that PES induced immunogenic cell death via lysosomal cathepsin D release in primary effusion lymphoma (12). In addition, PES enhanced 17AAG, an Hsp90 inhibitor of antitumor activities against acute leukemia and bladder cancer cells $(13,14)$. Furthermore, PES synergistically enhanced antitumor activity of hyperthermia against prostate cancer cells (15). However, little is known about the effect of PES on human lung cancer cells, and no study have been conducted to evaluate whether PES shows a chemosensitizing effect on human lung cancer cells.

We investigated the ability of PES to inhibit proliferation of NSCLC cell lines in vitro and in vivo, and further explored the underlying molecular mechanisms. In addition, we evaluated the antitumor effect of PES in combination with tumor necrosis factor-related apoptosis-inducing ligand (TRAIL). Our results suggest that PES can effectively inhibit cell proliferation and migration in human NSCLC cells. Furthermore, PES can induce G0/G1 phase cell cycle arrest and cell apoptosis via a caspase-dependent manner. Overexpression of Hsp70 in A549 cells attenuates the effect of PES on cell proliferation inhibition. Knockdown of Hsp70 by siRNA in A549 cells plays a potent synergistic effect on cell proliferation inhibition. In addition to reduction of p-AKT and p-ERK, PES sensitized NSCLC cell lines to TRAIL-induced cell proliferation inhibition and apoptosis via upregulation of DR4 and DR5. Finally, our results show that lung cancer xenografts in nude mice are efficiently inhibited by PES treatment.

\section{Materials and methods}

Cell lines and reagents. The human non-small cell lung cancer (NSCLC) cells (A549 and H460) purchased from the American Type Culture Collection (Manassas, VA, USA) were maintained in DMEM supplemented with $10 \%$ FBS (Gibco, Gaithersburg, MD, USA) at $37^{\circ} \mathrm{C}$ in a humidified atmosphere with $5 \% \mathrm{CO}_{2}$. The heat-shock protein 70 (Hsp70) inhibitor PES, (pifithrin- $\mu$ ), was purchased from Calbiochem (San Diego, CA, USA). Recombinant TRAIL was obtained from Invitrogen (Carlsbad, CA, USA). PES and recombinant TRAIL were dissolved in DMSO (Sigma, St. Louis, MO, USA) and PBS containing $0.1 \%(\mathrm{w} / \mathrm{v})$ bovine serum albumin (BSA), respectively.

Plasmids and cell transfection. The plasmid pSG5-Hsp70 was kindly provided by Professor X. Sun (School of Basic Medical Sciences, Wuhan University, Wuhan, China). Hsp70 siRNA and control siRNA were obtained from GenePharma (GenePharma, Shanghai, China). A549 cells were transiently transfected with pSG5-Hsp70 using X-tremeGENE HP DNA Transfection Reagent (Roche, Basel, Switzerland) according to the manufacturer's instructions. A549 cells were transiently transfected with Hsp70 siRNA or control siRNA using Oligofectamine (Invitrogen) according to the manufacturer's instructions.

Cell viability analysis. The cell viability was determined by the Cell Counting Kit-8 (CCK-8; Dojindo Laboratories, Kumamoto, Japan) assay. Briefly, A549 and H460 cells were incubated in 96 -well plates at a density of $5 \times 10^{3}$ per $100 \mu 1$ of culture medium overnight. After treated with indicated concentration of PES for 24 and $48 \mathrm{~h}, 10 \mu \mathrm{l}$ of tetrazolium substrate were added to each well of the plate. After incubation at $37^{\circ} \mathrm{C}$ for $1 \mathrm{~h}$, the absorbance was recorded at a wavelength of 450 nm using a microplate reader (EXL800; BioTek, Winooski, VT, USA). Each experiment was determined in triplicate and repeated at least three times.

Wound healing assay. A549 and H460 cells (5x105) were placed and culture in 6-well plates overnight. A sterile $10-\mu 1$ pipette tip was used to create wounds in 6-well plates. The cells were washed with PBS and cultured with fresh medium with or without PES. After incubation for further $48 \mathrm{~h}$, an inverted microscope was used to determine the wounds.

Transwell migration assay. Transwell migration assay was conducted in a Transwell chambers (Corning, New York, NY, USA) with a polycarbonate membrane (8- $\mu$ m polyester membrane filter pores). Cells were starved for $24 \mathrm{~h}$ prior to the experiment, and then $1 \times 10^{5}$ cells were seeded into the upper chamber with $100 \mu \mathrm{l}$ serum-free medium. The bottom chamber contained $500 \mu \mathrm{l}$ of medium containing $10 \%$ FBS to serve as a chemoattractant. Following incubation at $37^{\circ} \mathrm{C}$ for $48 \mathrm{~h}$, the cells adhering to the lower surface of the membrane were fixed, stained, and captured.

Flow cytometry analysis. Cell cycle arrest and induction of apoptosis by PES were assessed by flow cytometry (BD Biosciences). A549 and H460 cells (2x105/well) were seeded in 6-well plates and treated with vehicle control or PES $(20 \mu \mathrm{M})$ for $24 \mathrm{~h}$, respectively. For cell cycle analysis, above cells were collected and fixed with $500 \mu \mathrm{l}$ of $70 \%$ ethanol at $-20^{\circ} \mathrm{C}$ overnight. The fixed cells were washed with cold PBS 3 times. Cell cycle distribution was determined by using Cell cycle Detection kit (Multisciences, Hangzhou, China) according to the manufacturer's instructions. For apoptosis analysis, PES or vehicle control treatment of A549 and H460 cells were collected. Annexin V-FITC/propidium iodide (PI) Apoptosis Detection kit (Multisciences) was used to detect cell apoptosis induced by PES according to the manufacturer's instructions.

Caspase-3 activity assay. Caspase-3 activity assay kits from Beyotime (Shanghai, China) were used to detect caspase-3 activity. In brief, human NSCLC cells were treated with PES and TRAIL, either alone or in combination for $24 \mathrm{~h}$. The above cells were washed with cold PBS 3 times, followed by lysis buffer treatment on ice for $30 \mathrm{~min}$. After centrifuged at $12,000 \mathrm{~g}$ for $10 \mathrm{~min}$, cell lysate supernatant $(10 \mu \mathrm{l})$, assay buffer $(80 \mu \mathrm{l})$ and caspase-3 substrate (Ac-DEVD-pNA, $10 \mu \mathrm{l})$ were added to each well in a 96-well plate. The samples were further incubated at $37^{\circ} \mathrm{C}$ for $12 \mathrm{~h}$, and their optical density 
(OD) was detected at a wavelength of $405 \mathrm{~nm}$ using a microplate reader (BioTek, EXL800; BioTek). Each experiment was determined in triplicate and repeated at least three times.

Western blotting. Cells were lysed in RIPA lysis buffer (Beyotime) supplemented with $0.5 \%$ cocktail protease inhibitor (Roche). The cell lysates were centrifuged at $12,000 \mathrm{~g}$ for $10 \mathrm{~min}$ at $4^{\circ} \mathrm{C}$, and the supernatants were collected. Protein concentrations in the supernatants were measured according to the bicinchoninic acid method using bovine serum albumin as a standard. Equal amounts of proteins mixed with $5 \mathrm{X}$ loading buffer were subjected to $10 \%$ SDS-PAGE gels and transferred to PVDF membranes (Bio-Rad, Hercules, CA, USA). After blocking the membranes with $5 \%$ non-fat milk in TBST for $1 \mathrm{~h}$ at room temperature, the membranes were incubated with desired primary antibodies overnight at $4^{\circ} \mathrm{C}$. After $3 \times 5$ min washes in TBST, the membranes were incubated with corresponding horseradish-peroxidaseconjugated secondary antibodies for $1 \mathrm{~h}$ at room temperature. ECL systems (Bio-Rad) were used to detect expression of antibody-bound proteins.

The primary antibodies used in this study were as follows: GAPDH (10494-1-AP) from Proteintech (Peking, China), Vimentin (5741), cleaved caspase-3 (9664), cleaved caspase-9 (7237), Hsp70 (4872), Akt (9272), p-Akt (4060), ERK (9102), and p-ERK (4370) from Cell Signaling Technology (Cambridge, UK), p21 (sc-397), cyclin A (sc-751), CDK2 (sc-163), MMP9 (sc-21733), E-cadherin (sc-8426) cleaved PARP (sc-56196), DR4 (sc-8411), and DR5 (sc-166624) from Santa Cruz Biotechnology (Santa Cruz, CA, USA). The secondary antibodies were horseradish-peroxidase-conjugated secondary anti-mouse IgG (Kerui Tech, Wuhan, China) or anti-rabbit IgG (Kerui Tech).

In vivo xenograft model. Athymic female nude mice (6 weeks of age) were purchased from Beijing HFK Bioscience Co. Ltd. (Beijing, China) and housed under pathogen-free conditions. Animal care and use were approved by the Medical Ethics Committee of Wuhan University.

A549 cells $\left(1 \times 10^{7}\right)$ were suspended in Matrigel (BD Biosciences) and inoculated subcutaneously into the mice. Twelve mice bearing evident tumors were arbitrarily assigned to PBS control group and PES treatment groups (six mice per group). When tumors reached a size of $\sim 5 \times 5 \mathrm{~mm}^{2}$, mice were treated with either a single of intraperitoneal injection of PES $(20 \mathrm{mg} / \mathrm{kg})$ or PBS every two days. After 3-week treatment, mice were euthanized with carbon dioxide. Tumor burdens were evaluated by measuring body weight, tumor weight, and tumor volume. Tumor volume was determined as $0.5 \mathrm{x}$ length $\mathrm{x}$ width ${ }^{2}$. Tumor samples were collected and fixed in $10 \%$ neutral buffered formalin. Hematoxylin and eosin staining and immunohistochemistry for histological analysis of tumor samples were performed as described previously (16).

Statistical analysis. Data were expressed as the mean \pm standard deviation (mean $\pm \mathrm{SD}$ ). Statistical analysis was performed using SPSS software (version 19.0, SPSS, Chicago, IL, USA). The significance of the difference between two groups was determined using the Student's t-test. Values of $\mathrm{P}<0.05$ were considered statistically significant.

\section{Results}

PES reduces cell viability of human NSCLC cells. The chemical structure of PES is shown in Fig. 1A. To determine the effect of PES on cell viability of human NSCLC cells, A549 and $\mathrm{H} 460$ cells were exposed to vehicle control or a series of different PES concentration, ranging from 2.5 to $40 \mu \mathrm{M}$, for 24 and $48 \mathrm{~h}$, respectively. CCK- 8 assays were used to detect cell viability. As shown in Fig. 1B, PES induced a time- and dosedependent loss of viability in A549 cells, with 50\% inhibitory concentration $\left(\mathrm{IC}_{50}\right)$ of 44.9 and $25.7 \mu \mathrm{M}$ by a $24-$ and $48-\mathrm{h}$ PES treatment. Similar results were also found in $\mathrm{H} 460$ cells treated by a series of different PES concentration, and the $\mathrm{IC}_{50}$ values were calculated to be 40.1 and $24.3 \mu \mathrm{M}$ by a 24- and 48-h PES treatment (Fig. 1C). These results suggested that PES efficiently inhibited cell proliferation of A549 and H460 cells.

PES suppresses the migration of human NSCLC cells. Accumulating evidence has demonstrated that the metastasis activity of cancer cells is a critical mechanism for cancer mortality and invasion, which facilitated the development of cancer. To this end, the wound healing assay and Transwell migration assay were performed to study the effect of PES on migration of human NSCLC cells. When compared to the control group, PES suppressed the wound healing in A549 and H460 cells (Fig. 1D). To exclude the effect of cell proliferation on cell migration, Transwell migration assay was further used to evaluate effect of PES on cell migration. Similarly, the inhibitory effect of PES on cell migration was confirmed by the Transwell migration assay, in which the number of migrated A549 and H460 cells was significantly reduced after treatment with PES for $48 \mathrm{~h}$ (Fig. 1E). These results suggested that PES inhibited cell migration of human NSCLC cells.

PES induces cell cycle arrest in human NSCLC cells. To determine the mechanism by which PES inhibits human NSCLC cell proliferation, flow cytometry was used to analyze cell cycle contribution of A549 and H460 cells after PES $(20 \mu \mathrm{M})$ treatment for $24 \mathrm{~h}$. As shown in Fig. 2A, PES treatment markedly inhibited cell cycle progression in A549 and H460 cells, which was observed by a significant decrease in the percentage of cells in the $\mathrm{S}$ phase and concomitant increases in cells in the G0/G1 phase. When compared to the control, treatment of PES increased the fraction of G0/G1 from 32.8 to $54.1 \%$, decreased the fraction of S phase from 55.4 to $32.8 \%$ in A549 cells (Fig. 2B). Similarly, when compared to the control, PES increased the fraction of G0/G1 from 34.2 to $48.4 \%$, decreased the fraction of $\mathrm{S}$ phase from 44.6 to $33.5 \%$ in $\mathrm{H} 460$ cells (Fig. 2C). Notably, a marked change in percentage of cells in G2/M phase was not found in A549 and H460 cells (Fig. 2B and $\mathrm{C}$ ).

Consistent with the observation by flow cytometry, western blotting results indicated that PES inhibited expression of cyclin A and CDK2, which are cell cycle progression markers (Fig. 2D). In contrast, expression of CDK inhibitor p21, a negative regulator of cell cycle progression, was increased by PES treatment (Fig. 2D). Since inhibition of cell migration may be partly caused by inhibition of cell proliferation, western 
A<smiles>NS(=O)(=O)C#Cc1ccccc1</smiles>

Structure of PES
B

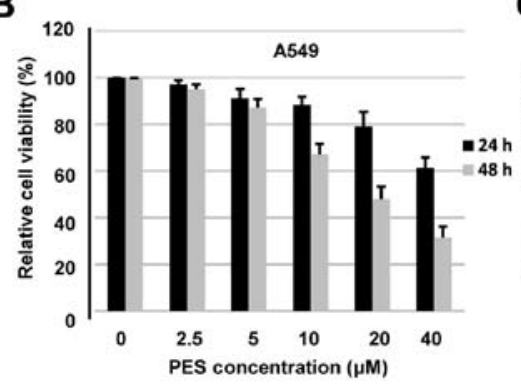

C

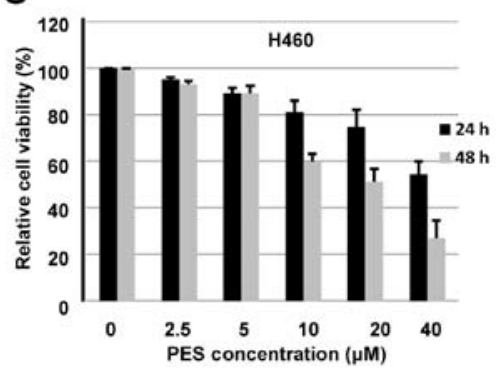

$\mathbf{E}$

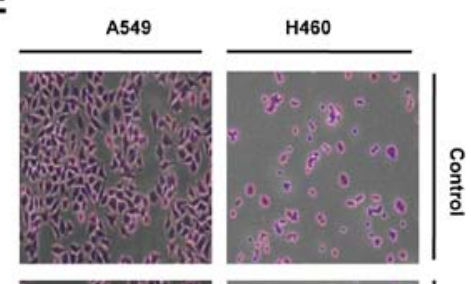

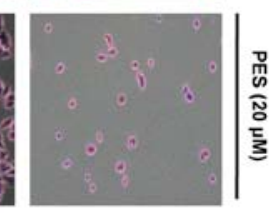

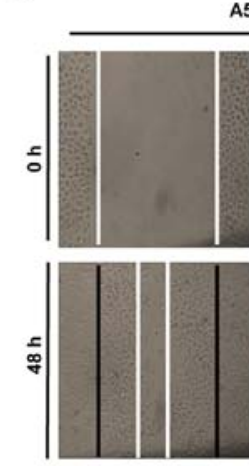

0
A549
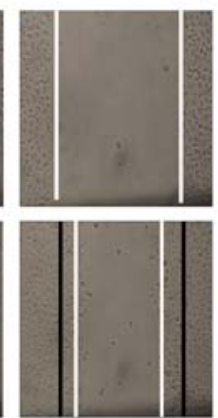

20

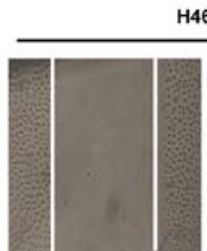

H460
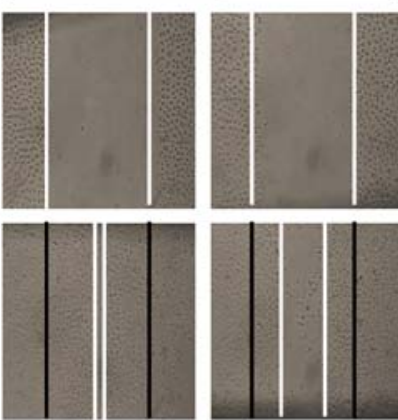

0

PES concentration $(\mu \mathrm{M})$

Figure 1. PES inhibits cell proliferation and migration of human NSCLC cells in vitro. (A) Sructure of PES. (B) A549 and (C) H460 cells were treated with vehicle control or a series of increasing concentrations of PES for 24 and $48 \mathrm{~h}$, respectively. The cell viabilities (\%) were measured by CCK- 8 assay. Results are shown as mean \pm SD of 3 independent experiments carried out in triplicate. (D) A549 and H460 cells were wounded using a 10- $\mu 1$ micropipette tip, after which they were further treated with or without PES $(20 \mu \mathrm{M})$ for $48 \mathrm{~h}$. The lines show the area where the scratch wound was created, and the images were captured using an inverted phase contrast microscope. (E) A549 and H460 cells were placed in Transwell chambers and incubated with or without PES (20 $\mu$ M) for $48 \mathrm{~h}$. Representative images of cells stained with crystal violet are shown at x100 magnification.

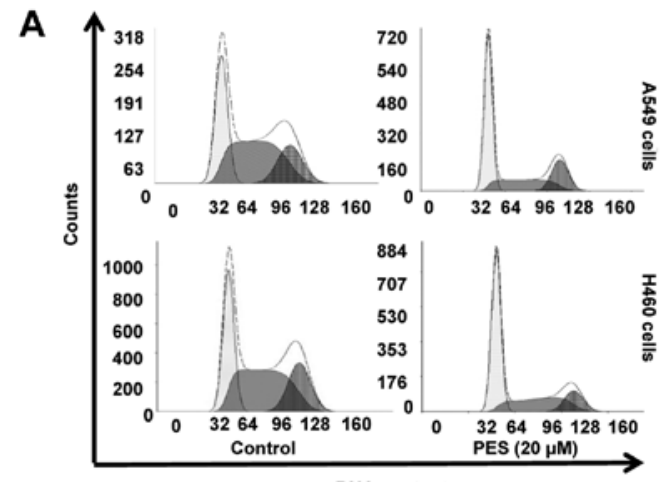

C

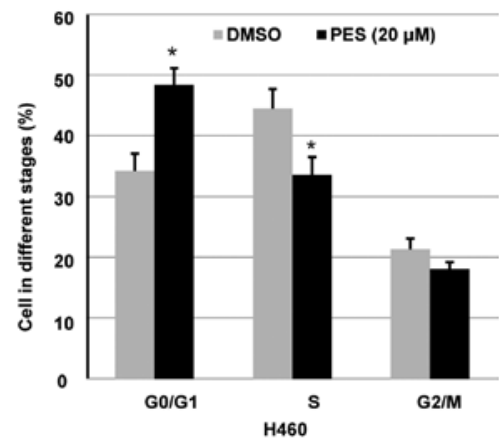

B

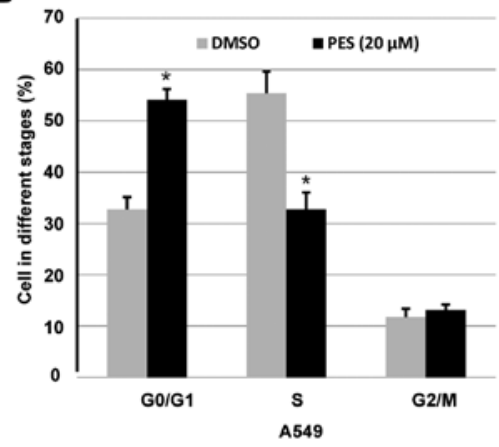

D

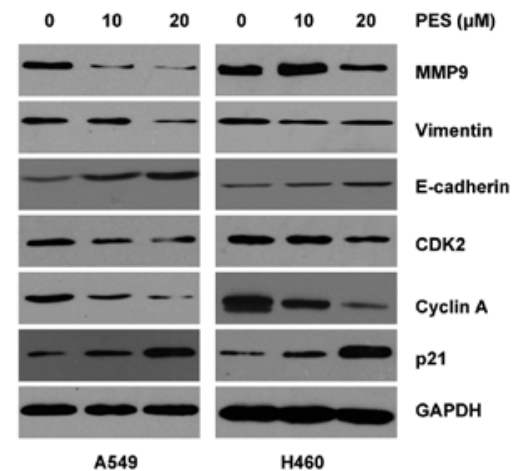

Figure 2. PES induces cell cycle arrest of human NSCLC cells. (A) Cell cycle analysis of vehicle control and PES treatment in human NSCLC cells. A549 and H460 cells were treated with vehicle control or PES $(20 \mu \mathrm{M})$ for $24 \mathrm{~h}$, respectively. After straining with PI, flow cytometry was used to detect contribution of the cell cycle. The cell cycle distributions in A549 (B) and H460 (C) treated by PES are quantified by the bar graphs. (D) A549 and H460 cells were treated with vehicle control or PES (10 or $20 \mu \mathrm{M})$ for $48 \mathrm{~h}$, whole cell extracts were collected and immunoblotted with anti-MMP-9, Vimentin, E-cadherin, CDK2, Cyclin A, and p21. GAPDH was used for loading control. 
A

A

B
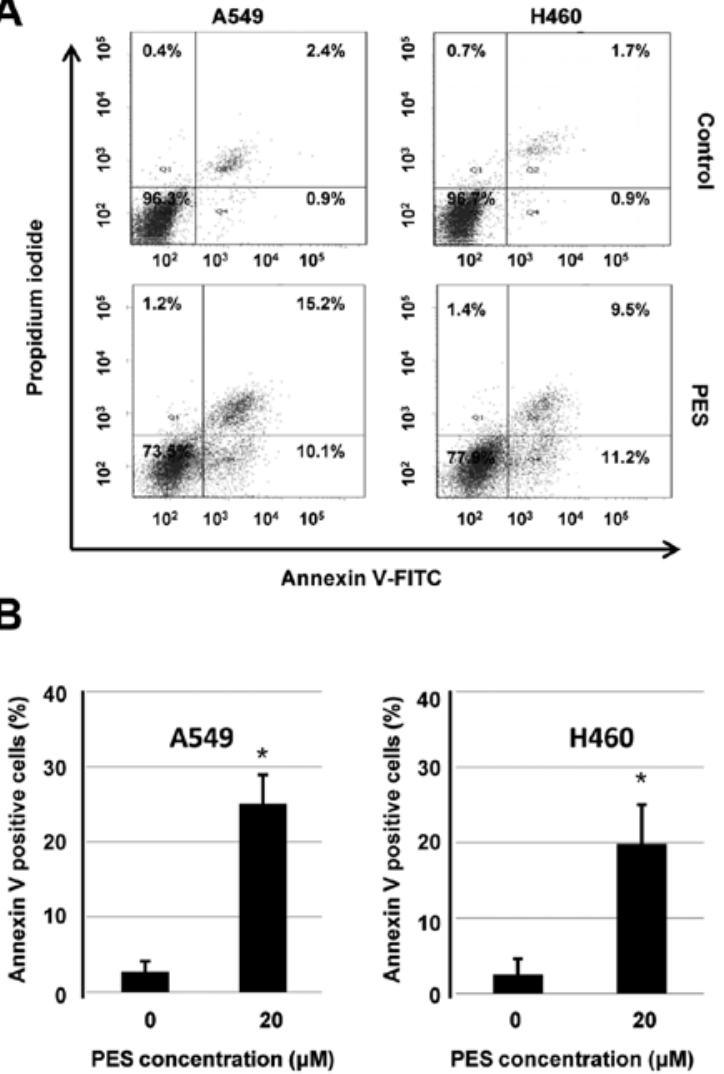

C

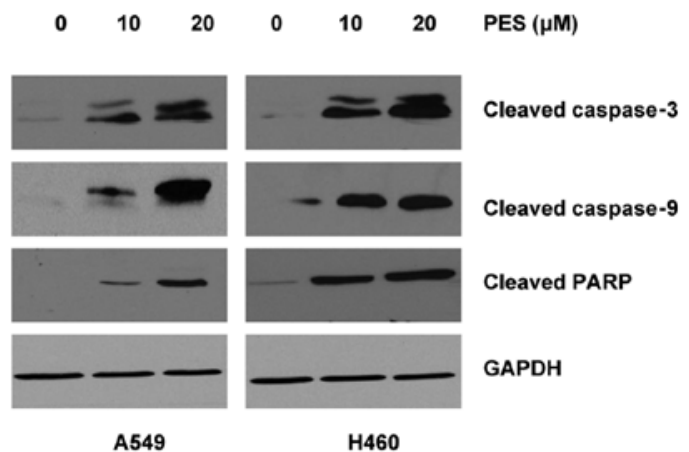

D

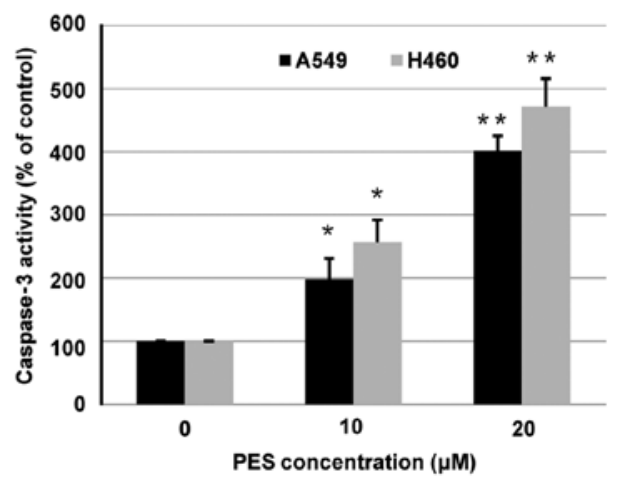

Figure 3. PES induces apoptosis of human NSCLC cells. (A) The effects of PES on induction of apoptosis. A549 and H460 cells were treated with vehicle control or PES $(20 \mu \mathrm{M})$ for $24 \mathrm{~h}$. After straining with Annexin V-FITC/PI, flow cytometry was used to detect cell apoptosis. (B) Annexin V-positive cells treated by PES for $24 \mathrm{~h}$ are quantified by the bar graphs. (C) A549 and H460 cells were treated with vehicle control or PES (20 $\mu \mathrm{M})$ for $48 \mathrm{~h}$, whole cell extracts were collected and immunoblotted with anti-cleaved caspase-3, anti-cleaved caspase-9, and anti-cleaved PARP. GAPDH was used for loading control (D) A549 and $\mathrm{H} 460$ cells were treated with vehicle control or PES (10 or $20 \mu \mathrm{M})$ for $24 \mathrm{~h}$, followed by lysis buffer treatment. Caspase-3 activity assay kits were used to evaluate effect of PES on apoptosis.

blotting was used to further detect the expression of genes associated with cell invasion. Consistent with the results of wound healing assay, PES increased expression of the epithelial marker E-cadherin and reduced expression of vimentin and MMP9, suggesting that PES inhibited human NSCLC cell migration and invasion via regulation of epithelial to mesenchymal transition (EMT) (Fig. 2D).

PES induces cell apoptosis in human NSCLC cells. To determine whether the inhibitory effect of PES on cell viability was associated with the induction of cell apoptosis, A549 and H460 cells were treated with or without PES $(20 \mu \mathrm{M})$ for $24 \mathrm{~h}$. Flow cytometry was used to analyze apoptosis induced by PES. As shown in Fig. 3A, the induction of apoptosis by PES was validated by increased percentage of Annexin V-positive cells. Quantitative analysis of Annexin V-positive cells induced by PES treatment is shown in Fig. 3B.

In addition to Annexin V-FITC assay, western blot analysis was used to evaluate the effect of PES on induction of apoptosis. A549 and H460 cells were treated with or without PES $(10$ or $20 \mu \mathrm{M})$ for $48 \mathrm{~h}$. Whole-cell extracts were harvested and subjected to western blotting. As shown in Fig. 3C, PES treatment increased expression of cleaved caspase-3, cleaved caspase-9, and cleaved PARP in both A549 and H460 cells. Furthermore, activities of caspase-3 were dose-dependently increased by PES treatment in A549 and H460 cells (Fig. 3D). These results suggested that PES induced apoptosis, at least in part, through a caspase-dependent intrinsic mitochondrial pathway in human NSCLC cells.

PES inhibits activation of Akt and ERK pathway in human NSCLC cells. AKT and MAPK signaling pathway is essential to the action of chemotherapeutic drugs in the regulation of cell cycle progression and apoptosis $(17,18)$. Previous reports have indicated that AKT and ERK are key modulators of cell proliferation, and activation of AKT and ERK by phosphorylation is associated with tumor aggressiveness and prognosis in NSCLC $(19,20)$. To determine the effect of PES on AKT and ERK signaling pathway, A549 and $\mathrm{H} 460$ cells were treated with vehicle control or PES $(10$ or $20 \mu \mathrm{M})$ for $48 \mathrm{~h}$. Results shown in Fig. 4A indicate that PES dose-dependently decreased phosphorylated AKT and ERK in A549 and H460 cells. In addition, DR4 and DR5 were increased following PES treatment.

Previous results have indicated that $\mathrm{Hsp} 70$ is essential to NSCLC growth regulation, and compared to normal counterpart cell lines, Hsp70 was overexpressed in NSCLC cells (5). Since PES is an Hsp70 inhibitor, the effect of PES on Hsp70 was evaluated in human NSCLC cells. As shown in Fig. 4A, PES did not reduce expression of Hsp70 in A549 
A

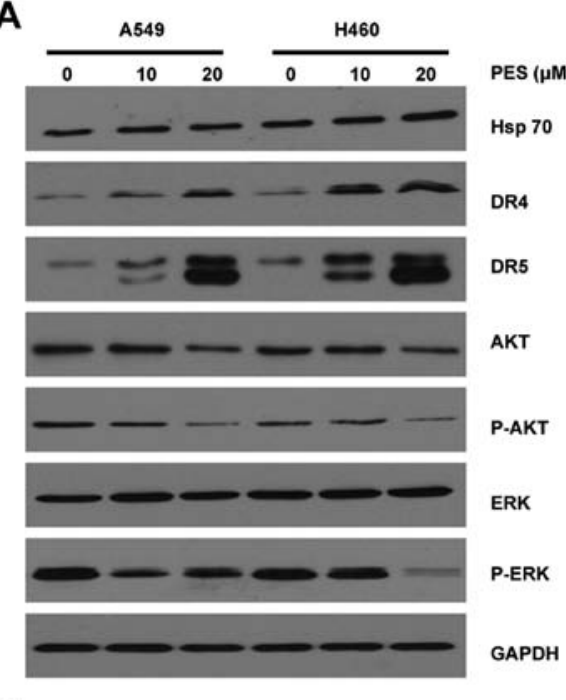

B

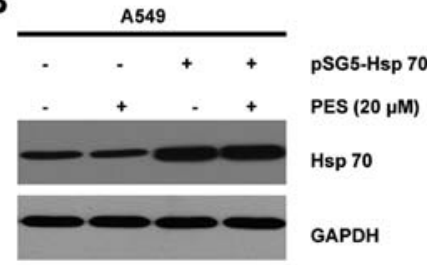

C

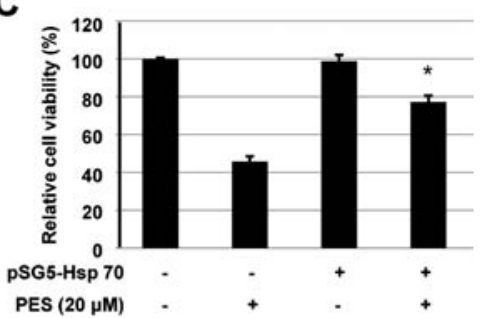

D

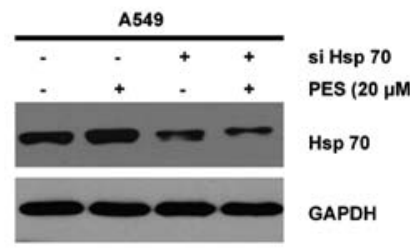

E

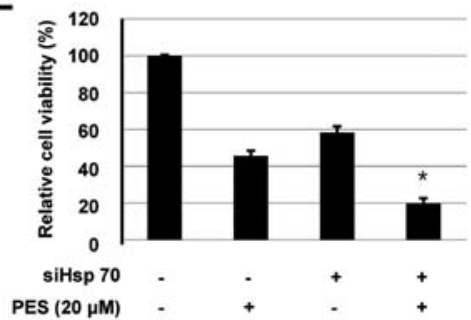

Figure 4. PES inhibits activities of AKT, ERK, and Hsp70 in human NSCLC cells. Effect of PES on activities of AKT and ERK. (A) A549 and H460 cells were treated with vehicle control or PES (10 or $20 \mu \mathrm{M})$ for $48 \mathrm{~h}$. Whole cell extracts were collected and immunoblotted with Hsp70, DR4, DR5, AKT, p-AKT, ERK, and p-ERK. GAPDH was used for loading control. (B and C) A549 cells transfected with or without pSG5-Hsp70 for 4 h were treated with or without PES for further $44 \mathrm{~h}$. Whole cells were collected and subjected to western blotting or CCK-8 assay as in Materials and methods above, respectively. (D and E) A549 cells transfected with or without siHsp70 for $4 \mathrm{~h}$ were treated with or without PES for further $44 \mathrm{~h}$. Whole cells were collected and subjected to western blotting or CCK-8 assay as in Materials and methods, respectively.

and $\mathrm{H} 460$ cells, suggesting that PES might inhibit activity of Hsp70 via specifically interacting with the ATPase binding domain.

PES-induced cell proliferation inhibition is involved in the regulation of $\mathrm{Hsp} 70$ in human NSCLC cells. To further validate if cell viability decrease by PES treatment is caused by acting on Hsp70, pSG5-Hsp70 or vehicle control (pSG5) were transiently transfected into A549 cells. At 4 h after transfection, cells were treated with or without PES $(20 \mu \mathrm{M})$ for further $44 \mathrm{~h}$. CCK-8 assay was used to detect cell viability after overexpression of Hsp70. As shown in Fig. 4B, Hsp70 was successfully overexpressed in A549 cells. Though overexpression of Hsp70 itself did not increase cell viability, it can efficiently attenuate the inhibitory efficiency of PES (Fig. 4C). To further characterize the role of Hsp70 in cell proliferation, Hsp70 was knocked down in A549 cells by siRNA. Results shown in Fig. 4D confirmed reduction of Hsp70 expression following by transfection with siHsp70. Moreover, Hsp70 knockdown by siHsp70 alone decreased cell viability by $58.8 \%$. Most importantly, A549 cells transfected with siHsp70 were more sensitive to PES-induced cell proliferation inhibition (Fig. 4E). These results suggested that PES inhibited cell proliferation of human NSCLS cells through Hsp70-dependent mechanism.

PES sensitizes NSCLC cell lines to TRAIL-induced cell proliferation inhibition and apoptosis. TRAIL belongs to a member of the tumor necrosis factor family that can trigger apoptosis in a broad spectrum of tumor cells while not in most normal cells (21). TRAIL can selectively trigger apoptosis of tumor cells through recognizing and binding to DR4 and DR5 on cell surfaces (22). Based on the upregulation of DR4 and DR5 in response to PES, we sought to investigate whether PES combined with TRAIL synergistically inhibits cell proliferation and enhance induction of apoptosis in NSCLC cells. A549 and $\mathrm{H} 460$ cells were treated with or without PES $(20 \mu \mathrm{M})$ in the absence or presence of TRAIL (40 ng/ml) for $24 \mathrm{~h}$, cell were harvested and subjected to cell viability, apoptosis, and caspase- 3 activation analysis, respectively.

As shown in Fig. 5A, PES alone and TRAIL alone reduced cell viability by 67.2 and $84.9 \%$, while co-treatment resulted in cell viability inhibition by $28.5 \%$ in A549 cells. Similarly, PES alone and TRAIL alone reduced cell viability by 59.9 and $66.1 \%$, while co-treatment resulted in cell viability inhibition by $19.7 \%$ in H460 cells (Fig. 5B). Flow cytometry results suggested that co-treatment with PES and TRAIL enhances cell apoptosis to a greater degree compared to PES treatment alone. Interestingly, TRAIL alone did not result in a marked induction of apoptosis in A549 and H460 cells. Quantitative analysis is shown in Fig. 5C and D. As expected, co-treatment of A549 and H460 cells with PES and TRAIL led to a marked increase in caspase- 3 activation, when compared to PES or TRAIL treatment alone. Results indicated in Fig. 5E demonstrate that treatment of cells with TRAIL alone did not induce the cleavage of PARP protein, while co-treatment of 
A

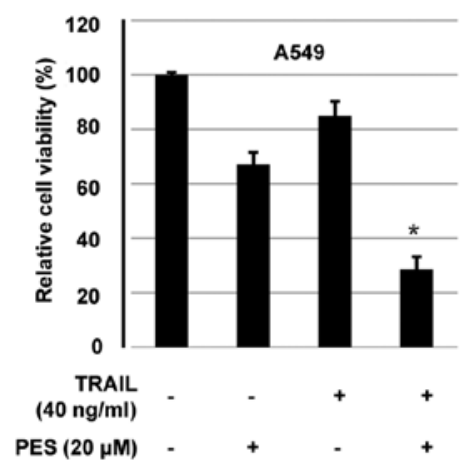

C

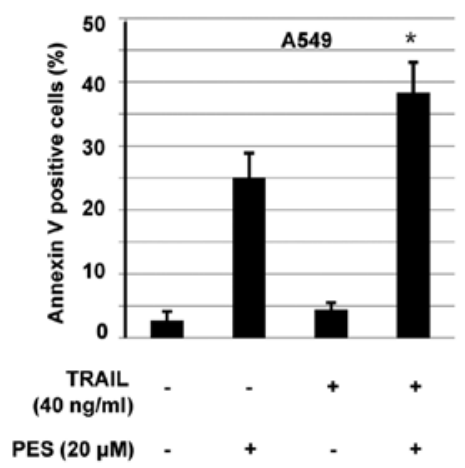

B

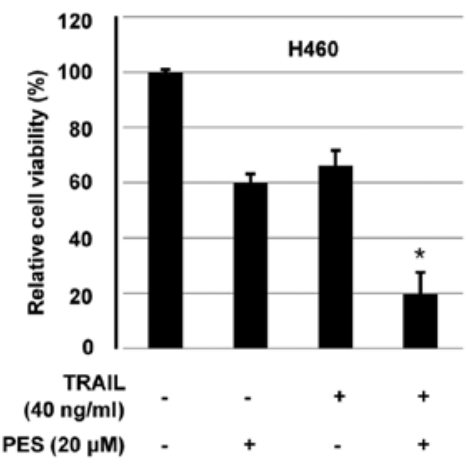

D

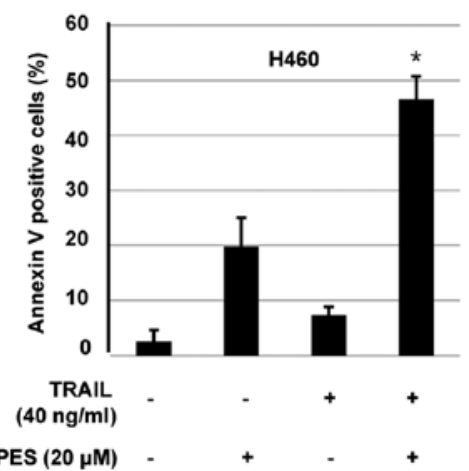

E

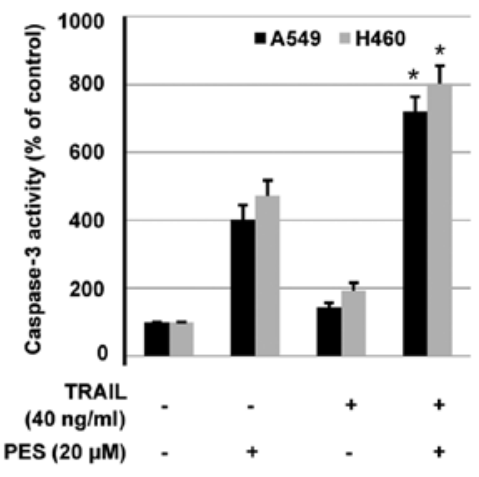

$\mathbf{F}$

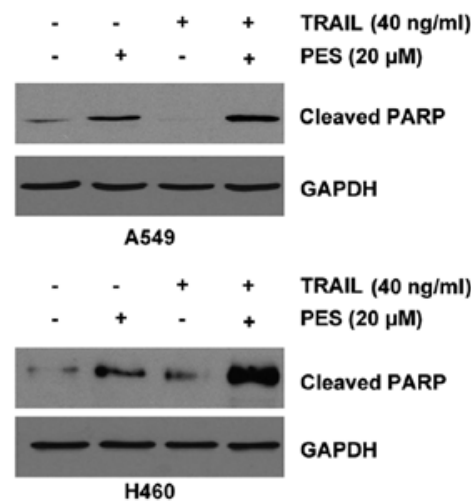

Figure 5. PES and TRAIL synergistically enhance cell proliferation inhibition and apoptosis in human NSCLC cells. (A) A549 and (B) H460 cells were incubated with or without PES in the absence and presence of TRAIL for $24 \mathrm{~h}$. The cell viabilities (\%) were measured by CCK-8 assay. Annexin V-positive cells for (C) A549 and (D) H460 cells treated as above are quantified by the bar graphs. (E) Caspase-3 activity for A549 and H460 cells treated as above using caspase-3 activity assay kits. (F) A549 and H460 cells were incubated with or without PES in the absence and presence of TRAIL for 48 h. Whole cell extracts were collected and subjected to western blotting for cleaved PARP and GAPDH.

cells with PES and TRAIL enhanced expression of cleaved PARP to a greater degree compared to PES treatment alone. Taken together, these results suggested that PES sensitized A549 and H460 cells to TRAIL-induced cell proliferation inhibition and apoptosis.

In vivo effects of PES on A549 lung tumor xenografts. To evaluate the antitumor activity of PES in a xenograft model, A549 cells $\left(1 \times 10^{7}\right)$ suspended in Matrigel were injected subcutaneously into flanks of female mice. After 7 days, mice bearing visible tumors $\left(\sim 5 \times 5 \mathrm{~mm}^{2}\right)$ were treated with PES $(10 \mathrm{mg} / \mathrm{kg})$ or PBS via intraperitoneal injection every two days. Three weeks later, the mice were euthanized, and tumors were measured and weighed. As shown in Fig. 6A and B, compared to PBS treatment, tumor volume and weight were reduced by PES treatment. Moreover, no significant mouse body weight loss was demonstrated in PES treatment groups (Fig. 5C).

Representative images of mice treated with either PBS or PES alone are shown in Fig. 6D. To further determine PES-associated toxicity in mice, major organs and tumors were dissected and subjected to hematoxylin and eosin staining and immunohistochemistry analysis. Results shown as Fig. 6E indicated that no notable differences between PES and PBS treatment group were observed among the organs involved, the liver, kidney, spleen, and lung. In addition, immunohistochemistry analysis results demonstrated that tumor tissues from the mice treated with PES also exhibited growth inhibition and increased apoptosis, as assessed by the staining for cleaved caspase-3, Ki-67, and TUNEL (Fig. 6F). Taken collectively, the above data suggested that PES exerts a potent in vivo inhibitory effect on lung carcinoma xenograft growth by inducing tumor cell apoptosis in mice. Moreover, PES used in this study made no marked signs of systemic toxicity, indicating that the dosage of PES in vivo in this study was safe.

\section{Discussion}

Over the past decades, lung cancer is one of the most commonly diagnosed cancers, and its morbidity and mortality have markedly increased in the world (23). Therefore, the effective agents and strategies for the treatment of lung cancer are urgently necessary. Cancer is known as a complex disease accompanying with multiple abnormally upregulated oncogenic proteins, which involve in activation of prosurvival signaling pathway (24). The chaperon function of Hsp family play an important role in the stability of most oncoproteins. Accumulating results have indicated that molecular chaperone function of Hsp family members is an attractive therapeutic target for the treatment of cancer $(6,25,26)$.

In this study, the biologic effects of a small molecular Hsp70 inhibitor, PES, on human NSCLC cells were determined in vitro and in vivo. First of all, PES were found to time- and 
A

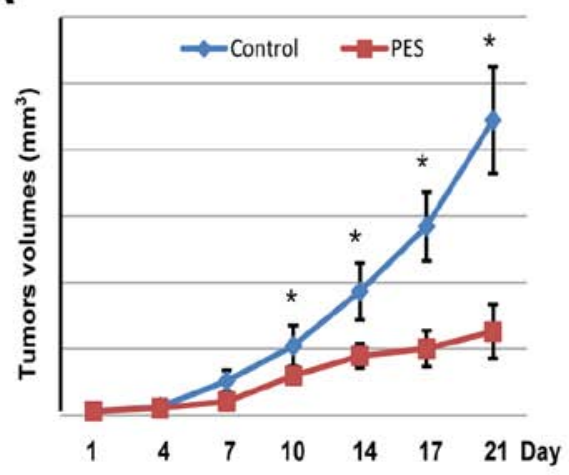

D
E
B

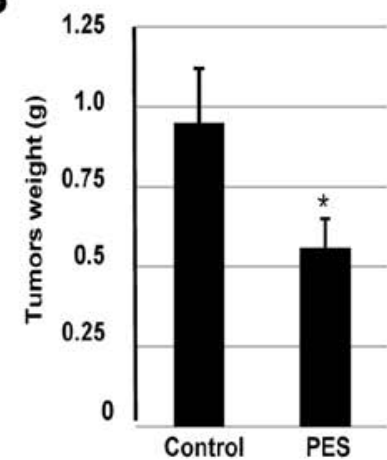

C

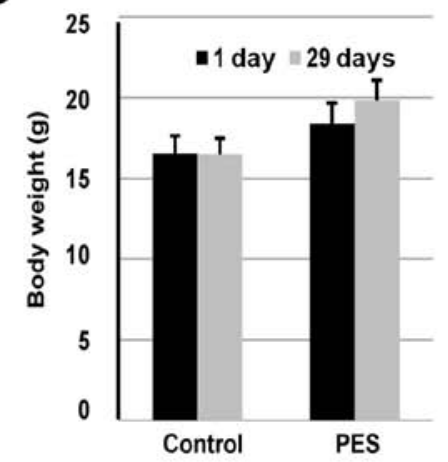
Control
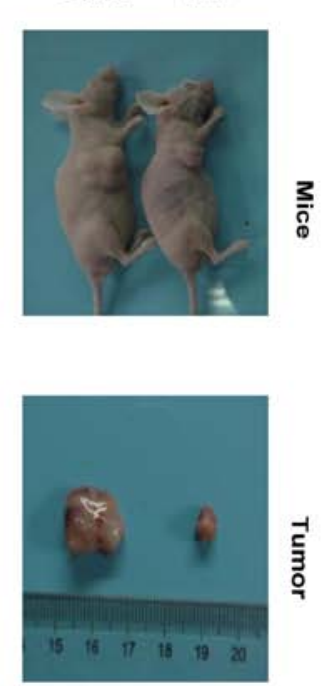

$\mathbf{F}$

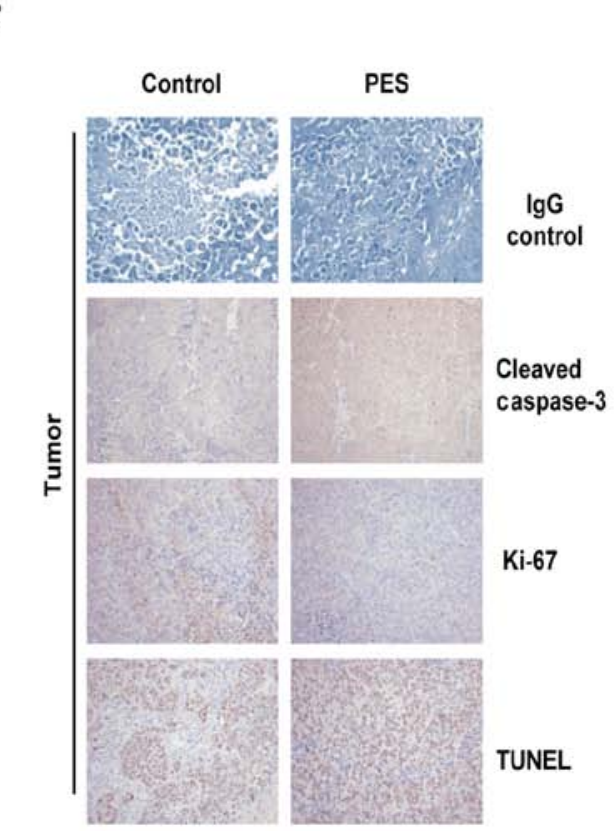

Figure 6. PES inhibits human NSCLC cells proliferation in vivo. Nude mice with established subcutaneous A549 tumors were treated with PBS or 20 mg/kg PES every two days for three weeks. (A) Tumor volumes, (B) tumor weight, and (C) body weight are shown as mean \pm SEM from each group. ${ }^{*}<0.05$, compared to control. (D) Representative images of mice and tumor from each group treated with either PBS or PES. (E) H\&E staining of major organs was performed to detect adverse systemic toxicity by PES in nude mice xenografted with A549 cells. (F) Immunohistochemical staining for tumors using TUNEL, anti-cleaved caspase-3, and anti-Ki-67.

dose-dependently inhibit cell proliferation in human NSCLC cells. Wound healing assay results indicated that compared to the vehicle control, wound width was significant wider after treatment by PES, suggesting that PES can efficiently inhibit cell migration of human NSCLC cells. Though inhibition of cell migration may be partly caused by inhibition of cell proliferation, Transwell migration assay results demonstrated that the number of migrated A549 and H460 cells was significantly reduced after treatment with PES for $48 \mathrm{~h}$, which confirmed the inhibition of cell migration by PES treatment. EMT is identified as a critical cellular phenomenon regulating tumor progression, and inhibition of EMT is considered a potent target for cancer therapy (27). The expression of E-cadherin, MMP-9, and Vimentin are related to cell invasion. Our results by western blotting demonstrated that PES reduced expression of MMP-9 and Vimentin, while increased expression of epithelial marker, E-cadherin. Therefore, PES inhibited human NSCLC cell migration at least in part via regulation of EMT.
In addition to inhibitory effect of growth, PES can induce cell cycle arrest and apoptosis. Flow cytometry results suggested that PES disrupted cell cycle progression, which was shown as a markedly increased percentage of NSCLC cells in G0/G1 phase. Furthermore, cell cycle progression markers, CDK2 and Cyclin A, were decreased, while CDK inhibitor, p21 was increased by PES treatments. The answer for the cell death through apoptotic or non-apoptotic pathway induced by PES is controversial. PES resulted in cell death in primary effusion lymphoma through induction of lysosomal cathepsin D release, not through canonical apoptotic processes (12). However, PES induced apoptotic cell death in pancreatic cell lines via both caspase-dependent and -independent processes (28). Our results demonstrated that PES induced cell apoptosis in human NSCLC cells. Caspase activation is the key event of apoptosis. Activated caspase-9 activates downstream caspase-3 and induces PARP cleavage (29). As expected, following treatment of human NSCLC cells by PES, expression of cleaved caspase- 9 was increased, accompanied by 
increased cleaved caspase- 3 and cleaved PARP. Furthermore, caspase- 3 activation assay results also confirmed the findings above, indicating that PES induced cell apoptosis, at least in part, in a caspase-dependent manner.

Multiple signaling pathways, such as ERK and AKT signal transduction pathways, play an important role in cell survival and regulation of apoptosis (30). Our results demonstrated that PES decreased the level of phosphorylated AKT and ERK in human NSCLC cells. These results concur with the reports in lung cancer cells treated with VER-155008 (5). In addition to protect cells from repeat exposure of harmful stimuli, Hsp70 can assist folding of nascent polypeptides and protein, indicating the importance of Hsp70 in growth and survival of tumor cells (31). Previous reports indicated that expression of Hsp70 was inhibited by triptolide, which resulted in pancreatic cancer cell death (6). Quercetin and gemcitabine synergistically enhanced apoptosis in lung cancer cells via inhibition of Hsp70 (32). Since PES is identified as an specific Hsp70 inhibitor, the regulation of Hsp70 by PES has been evaluated in human NSCLC cells. Though expression of Hsp70 was undetectably reduced by PES, overexpression of Hsp70 significantly attenuated the inhibitory effect of PES on cell proliferation in A549 cells, suggesting that PES targeted the ATPase binding domain of Hsp70, and then inhibited its protein chaperoning activity. As expected, knockdown of Hsp70 by siRNA in A549 cells enhanced sensitivity of PES to cell growth inhibition.

TRAIL, also referred as Apo- $2 \mathrm{~L}$, is an attractive antitumor therapeutic, which can selectively induce tumor cell apoptosis through binding to DR4 and DR5 in the cell surface (22). Since increased DR4 and DR5 by PES treatment were shown as the results above, the effects of combination treatment of PES and TRAIL on human NSCLC cells were determined. Our results indicated that combination treatment with PES and TRAIL synergistically inhibited cell proliferation and increased the percentage of Annexin V-positive cells in human NSCLC cells. Interestingly, TRAIL treatment alone in human NSCLC cells did not induce significant apoptosis, suggesting that human NSCLC cells presented resistance to TRAIL. The mechanisms of TRAIL resistance may contribute to the regulation of DRs, for increased DR4 and DR5 by PES treatment sensitized NSCLC cell lines to the effect of TRAIL. Even so, the specific mechanisms of TRAIL resistance to human NSCLC cells require further study.

In addition to the effect of PES on human NSCLC cells in vitro, this study also investigated the therapeutic effect of PES against NSCLC in vivo using a mouse xenograft model. Our results demonstrated that PES significantly repressed growth of tumor xenografts, which could be confirmed by decreased volume and weight of solid tumors. Moreover, that no notable differences were determined among mouse weight and major organs, suggesting that few side effects were observed by PES treatment in mice.

In conclusion, these results indicated the abilities of PES in vitro and in vivo to inhibit cell viability in human NSCLC cells. In addition, inhibition of cell viability by PES is related to the regulation of Hsp70 in human NSCLC cells. Though human NSCLC cells are resistant to TRAIL treatment, TRAIL showed a potent synergistic effects on cell proliferation inhibition via combination with PES. Taken together, PES may be a promising compound for the management of human NSCLC, and synergistic anticancer action of PES and TRAIL require further study.

\section{Acknowledgements}

This study was supported by the intramural funding from Jingzhou First People's Hospital of Hubei Province (to L.C.) and the research grant provided by JingZhou Hospital Affiliated to Huazhong University of Science and Technology.

\section{References}

1. Jemal A, Siegel R, Xu J and Ward E: Cancer statistics, 2010. CA Cancer J Clin 60: 277-300, 2010.

2. Erridge SC, Møller H, Price A and Brewster D: International comparisons of survival from lung cancer: Pitfalls and warnings. Nat Clin Pract Oncol 4: 570-577, 2007.

3. Jego G, Hazoumé A, Seigneuric R and Garrido C: Targeting heat shock proteins in cancer. Cancer Lett 332: 275-285, 2013.

4. Ritossa F: Discovery of the heat shock response. Cell Stress Chaperones 1: 97-98, 1996.

5. Wen W, Liu W, Shao Y and Chen L: VER-155008, a small molecule inhibitor of HSP70 with potent anti-cancer activity on lung cancer cell lines. Exp Biol Med (Maywood) 239: 638-645, 2014.

6. Phillips PA, Dudeja V, McCarroll JA, Borja-Cacho D, Dawra RK, Grizzle WE, Vickers SM and Saluja AK: Triptolide induces pancreatic cancer cell death via inhibition of heat shock protein 70. Cancer Res 67: 9407-9416, 2007.

7. Suzuki K, Ito Y, Wakai K, Kawado M, Hashimoto S, Seki N, Ando M, Nishino Y, Kondo T, Watanabe Y, et al: Serum heat shock protein 70 levels and lung cancer risk: A case-control study nested in a large cohort study. Cancer Epidemiol Biomarkers Prev 15: 1733-1737, 2006.

8. Huang Q, Zu Y, Fu X and Wu T: Expression of heat shock protein 70 and 27 in non-small cell lung cancer and its clinical significance. J Huazhong Univ Sci Technolog Med Sci 25: 693-695, 2005.

9. Zhuang H, Jiang W, Zhang X, Qiu F, Gan Z, Cheng W, Zhang J, Guan S, Tang B, Huang Q, et al: Suppression of HSP70 expression sensitizes NSCLC cell lines to TRAIL-induced apoptosis by upregulating DR4 and DR5 and downregulating c-FLIP-L expressions. J Mol Med (Berl) 91: 219-235, 2013.

10. Leu JI, Pimkina J, Frank A, Murphy ME and George DL: A small molecule inhibitor of inducible heat shock protein 70 . Mol Cell 36: 15-27, 2009.

11. Leu JI, Pimkina J, Pandey P, Murphy ME and George DL: HSP70 inhibition by the small-molecule 2-phenylethynesulfonamide impairs protein clearance pathways in tumor cells. Mol Cancer Res 9: 936-947, 2011.

12. Granato M, Lacconi V, Peddis M, Lotti LV, Di Renzo L, Gonnella R, Santarelli R, Trivedi P, Frati L, D'Orazi G, et al: HSP70 inhibition by 2-phenylethynesulfonamide induces lysosomal cathepsin D release and immunogenic cell death in primary effusion lymphoma. Cell Death Dis 4: e730, 2013.

13. Ma L, Sato F, Sato R, Matsubara T, Hirai K, Yamasaki M, Shin T, Shimada T, Nomura T, Mori K, et al: Dual targeting of heat shock proteins 90 and 70 promotes cell death and enhances the anticancer effect of chemotherapeutic agents in bladder cancer. Oncol Rep 31: 2482-2492, 2014.

14. Kaiser M, Kühnl A, Reins J, Fischer S, Ortiz-Tanchez J, Schlee C, Mochmann LH, Heesch S, Benlasfer O, Hofmann WK, et al: Antileukemic activity of the HSP70 inhibitor pifithrin- $\mu$ in acute leukemia. Blood Cancer J 1: e28, 2011.

15. Sekihara K, Harashima N, Tongu M, Tamaki Y, Uchida N, Inomata $T$ and Harada M: Pifithrin- $\mu$, an inhibitor of heat-shock protein 70, can increase the antitumor effects of hyperthermia against human prostate cancer cells. PLoS One 8: e78772, 2013.

16. Choi ES, Nam JS, Jung JY, Cho NP and Cho SD: Modulation of specificity protein 1 by mithramycin $\mathrm{A}$ as a novel therapeutic strategy for cervical cancer. Sci Rep 4: 7162, 2014.

17. Hers I, Vincent EE and Tavaré JM: Akt signalling in health and disease. Cell Signal 23: 1515-1527, 2011.

18. Dhillon AS, Hagan S, Rath O and Kolch W: MAP kinase signalling pathways in cancer. Oncogene 26: 3279-3290, 2007. 
19. Tang JM, He QY, Guo RX and Chang XJ: Phosphorylated Akt overexpression and loss of PTEN expression in non-small cell lung cancer confers poor prognosis. Lung Cancer 51: 181-191, 2006.

20. McCubrey JA, Steelman LS, Chappell WH, Abrams SL, Wong EW, Chang F, Lehmann B, Terrian DM, Milella M, Tafuri A, et al: Roles of the Raf/MEK/ERK pathway in cell growth, malignant transformation and drug resistance. Biochim Biophys Acta 1773: 1263-1284, 2007.

21. Walczak H, Miller RE, Ariail K, Gliniak B, Griffith TS, Kubin M, Chin W, Jones J, Woodward A, Le T, et al: Tumoricidal activity of tumor necrosis factor-related apoptosis-inducing ligand in vivo. Nat Med 5: 157-163, 1999.

22. Sprick MR, Weigand MA, Rieser E, Rauch CT, Juo P, Blenis J, Krammer PH and Walczak H: FADD/MORT1 and caspase- 8 are recruited to TRAIL receptors 1 and 2 and are essential for apoptosis mediated by TRAIL receptor 2 . Immunity 12: 599-609, 2000.

23. Jemal A, Bray F, Center MM, Ferlay J, Ward E and Forman D: Global cancer statistics. CA Cancer J Clin 61: 69-90, 2011.

24. Chan KC, Ting CM, Chan PS, Lo MC, Lo KW, Curry JE, Smyth T, Lee AW, Ng WT, Tsao GS, et al: A novel Hsp90 inhibitor AT13387 induces senescence in EBV-positive nasopharyngeal carcinoma cells and suppresses tumor formation. Mol Cancer 12: 128, 2013.

25. Chen W, Sin SH, Wen KW, Damania B and Dittmer DP: Hsp90 inhibitors are efficacious against Kaposi sarcoma by enhancing the degradation of the essential viral gene LANA, of the vira co-receptor EphA2 as well as other client proteins. PLoS Pathog 8: e1003048, 2012.
26. Wang J, Li Z, Lin Z, Zhao B, Wang Y, Peng R, Wang M, Lu C, Shi G and Shen Y: 17-DMCHAG, a new geldanamycin derivative, inhibits prostate cancer cells through Hsp90 inhibition and survivin downregulation. Cancer Lett 362: 83-96, 2015.

27. Baek SH, Ko JH, Lee JH, Kim C, Lee H, Nam D, Lee J, Lee SG, Yang WM, Um JY, et al: Ginkgolic acid inhibits invasion and migration and TGF- $\beta$-induced EMT of lung cancer cells through PI3K/Akt/mTOR inactivation. J Cell Physiol 232: 346-354, 2017.

28. Monma H, Harashima N, Inao T, Okano S, Tajima $\mathrm{Y}$ and Harada M: The HSP70 and autophagy inhibitor pifithrin- $\mu$ enhances the antitumor effects of TRAIL on human pancreatic cancer. Mol Cancer Ther 12: 341-351, 2013.

29. Ding X, Zhang B, Pei Q, Pan J, Huang S, Yang Y, Zhu Z, Lv Y and Zou X: Triptolide induces apoptotic cell death of human cholangiocarcinoma cells through inhibition of myeloid cell leukemia-1. BMC Cancer 14: 271, 2014.

30. Meng G, Wang W, Chai K, Yang S, Li F and Jiang K: Combination treatment with triptolide and hydroxycamptothecin synergistically enhances apoptosis in A549 lung adenocarcinoma cells through PP2A-regulated ERK, p38 MAPKs and Akt signaling pathways. Int J Oncol 46: 1007-1017, 2015.

31. Zorzi $\mathrm{E}$ and Bonvini P: Inducible hsp70 in the regulation of cancer cell survival: Analysis of chaperone induction, expression and activity. Cancers (Basel) 3: 3921-3956, 2011.

32. Lee SH, Lee EJ, Min KH, Hur GY, Lee SH, Lee SY, Kim JH, Shin C, Shim JJ, In KH, et al: Quercetin enhances chemosensitivity to gemcitabine in lung cancer cells by inhibiting heat shock protein 70 expression. Clin Lung Cancer 16: e235-e243, 2015. 